\title{
Design and control of precision work stage with air bearing for large size optical grating fabrication
}

\author{
SIRIGE $^{1, \mathrm{a}^{*}}$ \\ ${ }^{1}$ Mechanical engineering, Beijing University of technology, Chaoyang, Beijing, China
}

\begin{abstract}
Scanning Beam Interference Lithography (SBIL) is an advanced large-diameter and high-precision plane grating manufacturing solution, which has very high requirements for the positioning accuracy and running speed of the work stage carrying the substrate. In order to improve the size and accuracy of optical grating manufacturing in scanning interference lithography further, air bearing with high precision, high loading capacity and high stiffness is one of the key components for the work stage. In this paper, in order to design a large stroke work stage which meets the demand, firstly, the air bearing, the core part of the work stage, is analyzed and calculated by finite element method, and the pressure distribution curve of the air bearing of the work stage is obtained. On this basis, the linear air bearing with high bearing capacity and high stiffness is designed by using the omni-directional preloading analysis method of high-precision motion platform. Based on the high-precision position feedback of grating encoder, the high-precision closed-loop control of $20 \mathrm{~nm}(3 \sigma)$ is realized by using PID control and trap filter, which meets the design and requirements, and provides technical and engineering support for the manufacture of large-scale optical grating.
\end{abstract}

\section{Introduction}

Scanning Beam Interference Lithography is a new manufacturing technology of large area optical grating in recent years. Its basic principle is to use two beams of coherent light to form an interference field, continuously expose the substrate fixed on the uniform motion stage, and obtain large size optical grating by dynamic scanning and step splicing ${ }^{[1]}$. In order to achieve grating manufacturing with nanometer precision, the positioning accuracy and trajectory accuracy of the work stage are very high. The air-bearing work stage has the advantages of no friction, low damping and so on. At the same time, it adopts the direct drive technology of linear motor, which simplifies in structure, higher in accuracy and efficiency than the traditional rotary motor and screw guide.

The one-dimensional precision air-bearing work stage developed by Zhu Yu's team of Tsinghua university can achieve a dynamic accuracy of $\pm 60 \mathrm{~nm}$ in the stroke range of more than $120 \mathrm{~mm}^{[2]}$; Chen et al. used the spring element to simulate the stiffness model of air bearing, and proposed the design method of air bearing support stiffness of precision air bearing stage ${ }^{[3]}$; CAI's team equivalent the air bearing to the design link of linear and torsion spring for air bearing stage Parameter guidance and correction ${ }^{[4]}$; Zhang $\mathrm{C} \mathrm{P}$ and Liu Q used the finite element analysis method to simulate and optimize the dynamic characteristics of the air bearing ${ }^{[5]}$; Chen P F and Wang Y W based on Model Center to model and optimize the parameters of the air bearing stage ${ }^{[6]}$. The above methods are designed and simulated by the spring equivalent of the air bearing. These methods have a large gap with the actual working conditions. Once there are nanometer precision requirements for the worktable, these methods will no longer meet the design requirements.

In this paper, the air-bearing work stage of is taken as the research object, and the development and application of H-type work stage driven by linear motor is taken as the purpose. First, the pressure distribution in the air film of the air bearing is analyzed, and based on the finite element method, different preloading techniques are used to simulate the bearing capacity of the air bearing, which leads to the design requirements of high bearing capacity and high stiffness. Then, the overall design of the work stage which can be applied to the SBIL is completed. Finally, aiming at the synchronous control problem of the bilateral linear motor of the work stage, the traditional parallel synchronous control method is adopted. In order to ensure the closed-loop stability of the motion platform, the controller adopts stable PID control. On this basis, the trap filter is added to suppress the mechanical resonance, and the lead link is added to realize the phase lead and ensure the phase margin.

\section{Design of air bearing for work stage}

\subsection{Design requirement analysis}

In this paper, we plan to design a precision air-bearing work stage, which is mainly supported by air bearing and

* Corresponding author: ${ }^{\mathrm{a}} \mathrm{srg}$ Alashan@ $163 . c o m$ 
driven by linear motor. In order to realize the manufacturing of large-scale optical grating with nanometer precision, the work stage on the substrate is required to have $\mathrm{XY}$ two degrees of freedom, in which $\mathrm{X}$ axis is the scanning axis, Y-axis is the stepping axis, and the grating encoder is used to provide nanometer precision positioning as the measurement feedback guarantee the position accuracy of the work stage is $20 \mathrm{~nm}$.

\subsection{Finite element calculation method of air bearing}

The design of air bearing refers to the design of externally pressurized air bearing under the working condition of low and medium speed and bearing lubrication mainly based on static pressure effect. It mainly focuses on the static load, static stiffness and friction power consumption of air bearing under stable working condition.

The commonly used engineering calculation method is to obtain the analytical solution of Reynolds equation through simplified empirical formula, but the calculation error of static characteristics of air bearing is large, which cannot be calculated. For the air bearing with complex structure shape and flow state, it cannot be used to determine the pressure distribution. Therefore, the finite element method is used to solve the discretization of air film pressure field directly based on Reynolds equation Then, the static design of the air bearing is completed by ANSYS. The structure and finite element mesh of the orifice throttling air bearing are shown in Figure 1.
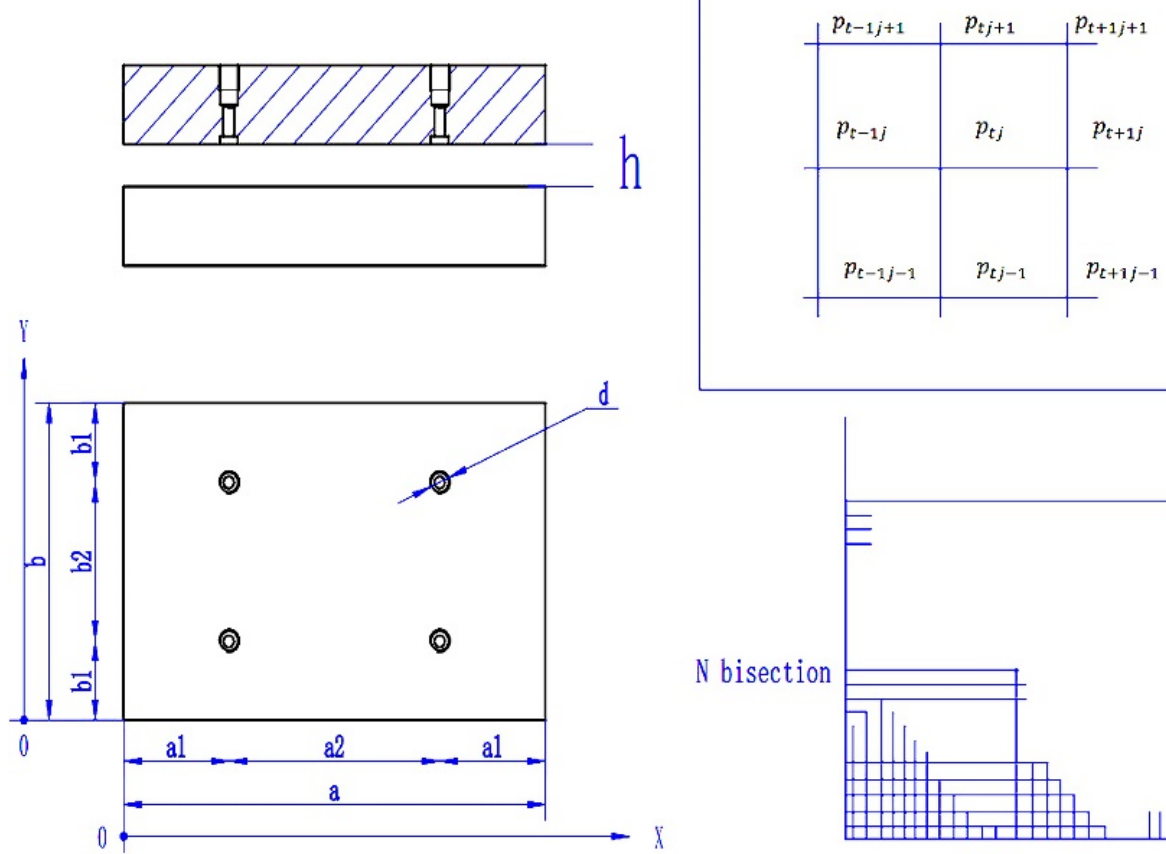

Figure 1 Air bearing and its meshing

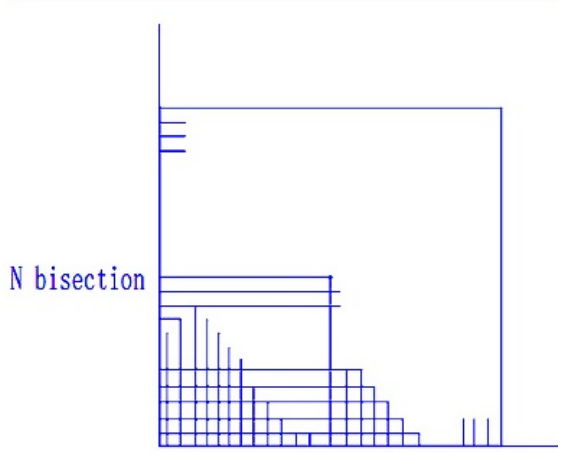

M bisection
According to the assumption that the gas in the film is isothermal and steady, the Reynolds equation of the flowing compressed gas is simplified as follows:

$$
\frac{\partial^{2} p^{2}}{\partial x^{2}}+\frac{\partial^{2} p^{2}}{\partial y^{2}}=D
$$

Because of the air environment around the air bearing, its boundary condition is:

$$
\left\{\begin{array}{l}
\text { when } x=0, x=a, p=p_{a} \\
\text { when } y=0, y=a, p=p_{a}
\end{array}\right.
$$

Where $p_{a}$ is the ambient gas pressure.

According to the boundary conditions of the gas bearing shown in equation (2), equation (1) can be solved to simplify the discrete solution of Reynolds equation the pressure distribution in the film. Then, according to the pressure distribution, the static parameters of gas bearing, such as bearing capacity, stiffness, flow rate and film thickness, are solved. The flow chart of finite element calculation of air bearing guide rail is shown in Figure 2.

The parameters in Figure 2 show that $p_{o}$ is the outlet pressure of the orifice, $p_{s}$ is the supply pressure, $W$ is the bearing capacity, $h$ is the film thickness, $w_{\text {in }}$ is the amount of gas flowing into the air bearing, and $w_{\text {out }}$ is the amount of gas flowing out of the air bearing.

The calculation process shown in Figure 2 integrates the engineering calculation method and finite element calculation method. With this development tool, a fourorifice bearing of the work stage is calculated, and the pressure values of each node of the finite element are drawn into a plan. The two-dimensional distribution of the gas film pressure field is shown in Figure 3.

Figure 3 shows that the gas film pressure field of thrust bearing with orifice throttling is octagonal distribution, the pressure at orifice is the highest, and the edge pressure of air bearing is equal to the external environment pressure, so the air bearing has relatively low angular stiffness. In the design of air bearing, the position of orifice should be set reasonably to obtain a satisfied angular stiffness. 


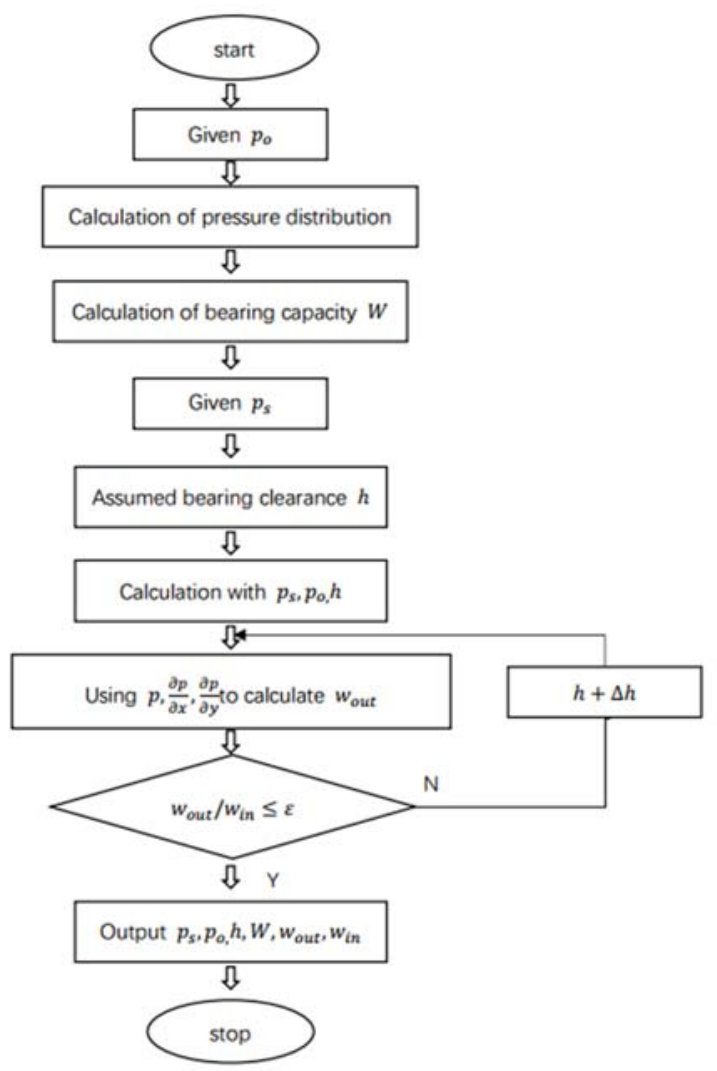

Figure 2 Flow chart of finite element calculation for air bearing

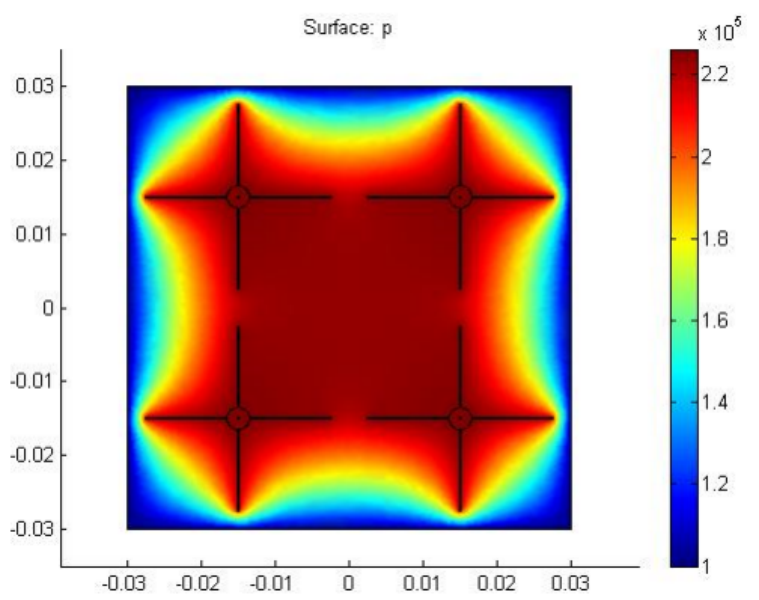

Figure 3 Two-dimensional distribution of air film pressure field

\subsection{Preloading technology of air bearing}

Preloading technology is an effective way to improve the bearing capacity and stiffness of air bearing. There are three main methods: air film preloading, vacuum preloading and magnetic preloading. Air film preloading is to design two opposite air films in the support direction, so as to produce preload, so as to make it have the capacity of bearing two-way load and two-way stiffness, and the stability of air bearing is better.

Vacuum preloading is to design a vacuum chamber on the air floating working surface of the slider. The slider is absorbed on the air floating surface by vacuum negative pressure, so as to generate preload and improve the stiffness of the air bearing. When the buoyancy and suction are balanced, a stable working air film is formed. The technology of vacuum preloading is complex.

The magnetic preloading is that permanent magnets such as magnetic steel are embedded on the guide rail and sliding sleeve, and the slider is absorbed on the air floating surface by the magnetic attraction to form the magnetic preloading. Magnetic preloading technology is simple in structure and easy to adjust.

The work stage designed in this paper mainly adopts two kinds of Technology: air film preloading and magnetic preloading.

\section{Structural design of work stage}

Using the finite element calculation method and preloading technology, the linear motor H-type air-bearing work stage is designed by using the air bearing calculation software designed in this paper, as shown in Figure 4.

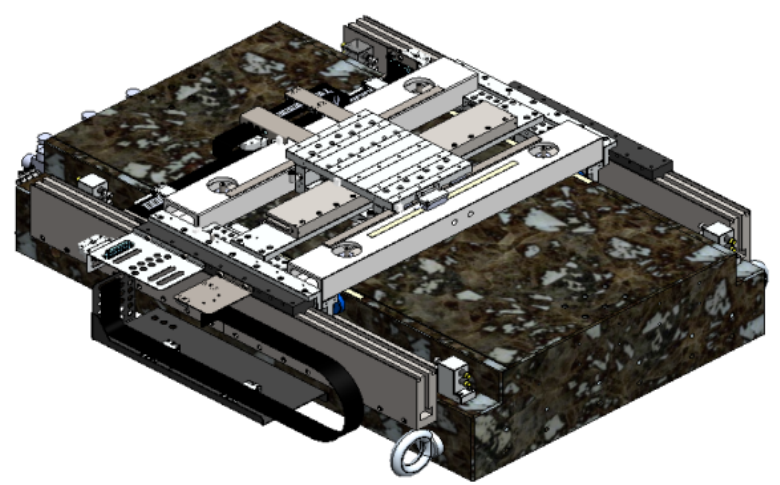

Figure 4 H-type air-bearing work stage

\subsection{Main structure design}

The XY axis of the work stage shown in Figure 4 adopts the structure of H-type dual drive. The core part of the $y$ axis movement is the ceramic beam frame, and the $\mathrm{x}$-axis slider moves in the ceramic beam frame, realizing the movement of X and Y degrees of freedom. The base of work stage system is made of granite with low thermal expansion coefficient and high dimensional stability. The scheme embodies the advantages of linear motor and air bearing, realizes no friction and zero transmission, and ensures the high precision and high dynamic response of the motion system.

The Closed structure has the characteristics of high precision, high rigidity and large load. Therefore, the yaxis horizontal direction adopts closed air film preloading, and the vertical direction adopts open magnetic preloading. In the same horizontal direction, $\mathrm{x}$-axis adopts closed air film preloading, and in the same vertical direction adopts open magnetic preloading. The $\mathrm{x}$-axis and $\mathrm{y}$-axis adopt all direction preloading design to obtain satisfied bearing capacity and stiffness.

The $y$-axis should bear the mass of all the parts of the two-dimensional air floating table, and at the same time, it should overcome the overturning moment of the $\mathrm{x}$-axis in the feeding process. In order to meet the work needs of greater load-carrying capacity, so y-axis choose double motor drive, the grating encoder feedback, vertical 
direction adopts open air flotation and magnetic preloading technology, horizontal direction adopts closed air flotation, in order to enhance the stiffness which will adopt air film preloading technology.

In the laser processing of large-size grating, the stage is not affected by cutting force, and the $\mathrm{x}$-axis only bears the weight of the stage and substrate, so the requirements of bearing capacity and stiffness are low. In order to avoid interference with the y-axis, make the stage more compact and lower the center of gravity, the $\mathrm{x}$-axis is driven by a single motor, the grating encoder feedback, and the horizontal direction is closed air floating the vertical direction adopts open air floating magnetic preloading, adopts fully closed air floating structure, the structural frame adopts ceramic beam, and the bottom surface adopts granite as air floating surface.

\subsection{Static characteristic test of air bearing}

Static stiffness test of $\mathrm{x}$-axis vertical direction: experimental gas supply pressure $P_{s}=5 \mathrm{bar}$, vacuum chamber pressure $P_{v}=0.6 \mathrm{bar}$. The relationship between air floating clearance, load and stiffness curve in four orifice thrust bearing is shown in Fig. 5. The static stiffness of $\mathrm{x}$-axis in vertical direction is $103.7 \mathrm{~N} / \mu \mathrm{m}$, the static stiffness in horizontal direction is $213.6 \mathrm{~N} / \mu \mathrm{m}$, and the bearing capacity is $602 \mathrm{~N}$. It can meet the requirement of high-precision plane grating manufacturing with $170 \mathrm{~mm} * 170 \mathrm{~mm}$ and $5 \mathrm{~kg}$ glass-ceramic.

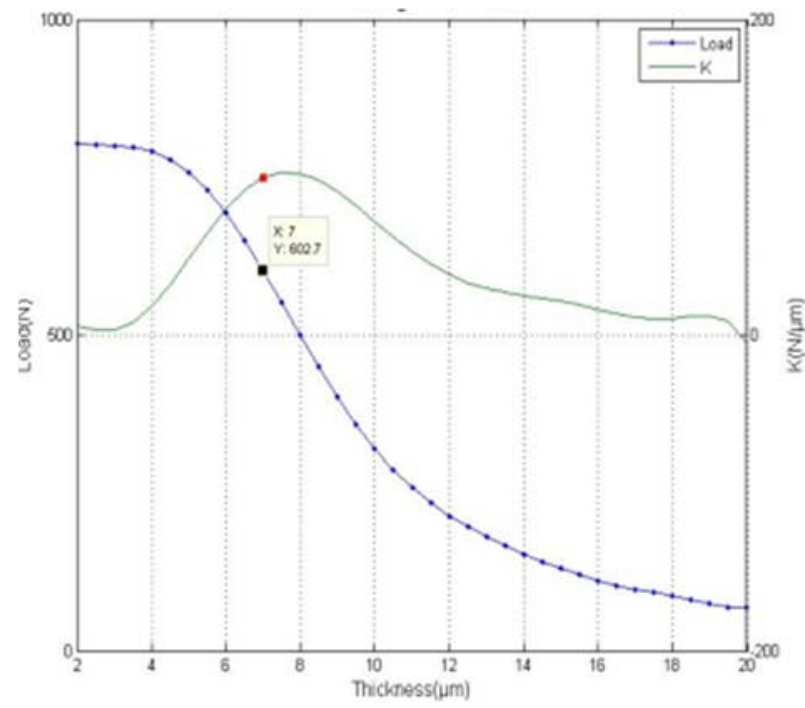

Figure 5 Relationship between air floating clearance and load, stiffness curve

\section{Model identification and control of work stage}

In the design of work stage control system, due to the deviation between the theoretical model and the actual model, it is necessary to obtain the work stage model which can be used for control by the method of model identification. In the closed-loop condition, the model of each degree of freedom is identified, and then the controller is designed according to the characteristics of the identified model. Input $100 \sim 1500 h z$ excitation signal under closed-loop condition, and get the identification model results as shown in Figure 7.

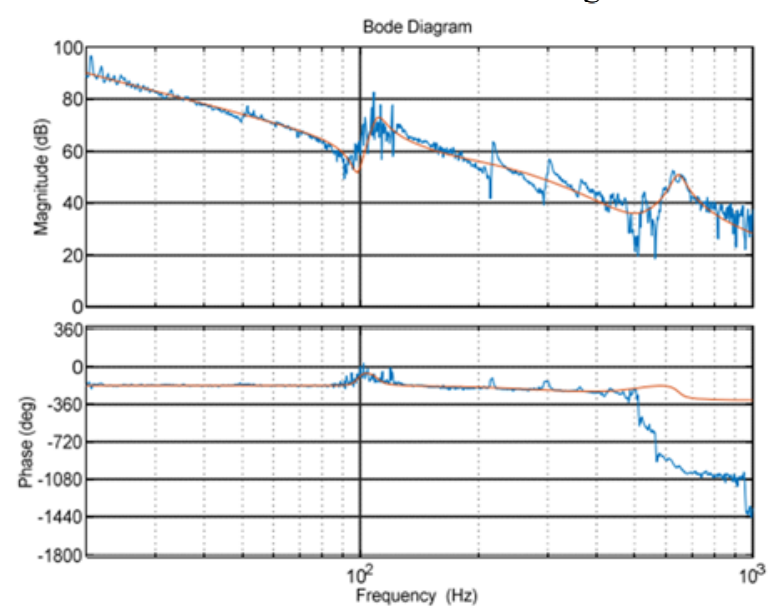

(a)

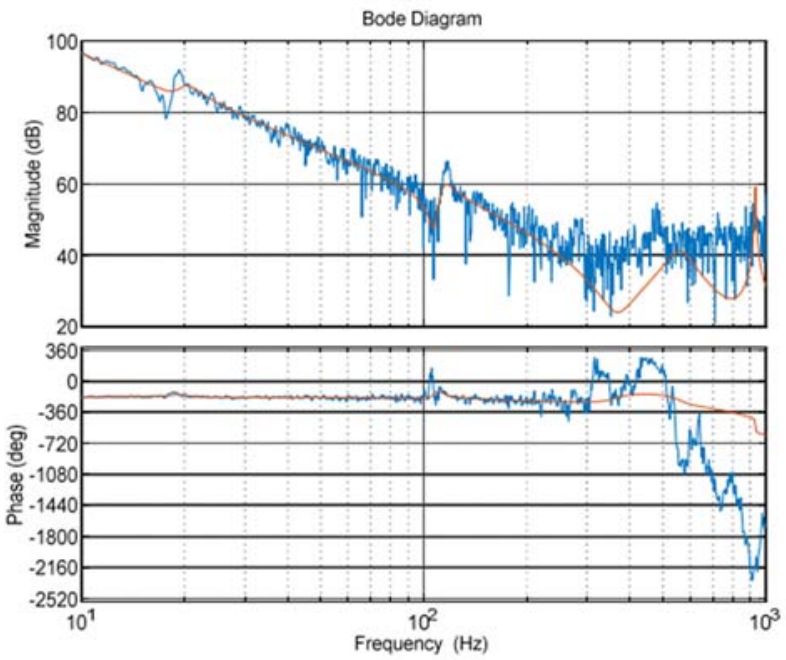

(b)

Figure 6 Identification and fitting results: (a) x-dof; (b) y-dof;

It can be seen from Figure 7 (a) that the amplitude frequency characteristics of the system below $400 \mathrm{~Hz}$ are close to the ideal double integral model, but the phase frequency curve has a large phase lag. Because the system is a rigid structure and has a large load, there is a large time lag. Here, the identification result is fitted by using the eighth order parameter model, and the fitting result is shown by the dotted line in Figure 7 (a). The fitting model is basically the same as the identification model It can be directly used in controller design.

In order to ensure the closed-loop stability of x-dof, the PID controller structure is adopted. On this basis, the trap filter is added to suppress the mechanical resonance, and the lead link is added to achieve the phase lead and ensure the phase margin.

Using the above method, the XY DOF controllers are designed respectively, and the control test is carried out on the stage system. As shown in the fitting curve in Fig. 7 (a), the cut-off frequency of the controller designed for $\mathrm{x}$ dof is $23.5 \mathrm{hz}$, the amplitude margin is $12.4 \mathrm{db}$, and the phase margin is $54^{\circ}$. As shown in the fitting curve in Fig. 7 (b), the controller designed for y-dof achieves a cut-off frequency of $19.8 \mathrm{hz}$, an amplitude margin of $18.2 \mathrm{db}$, 
and a phase margin of $47^{\circ}$ to ensure sufficient stability margin and achieve high control bandwidth, and effectively suppress the resonance peak.

The positioning error of each degree is shown in Figure 8 . The y-dof positioning accuracy determines the measurement accuracy of the interference fringe period, and the positioning accuracy in this direction can reach $16 \mathrm{~nm}$, which meets the requirements of the proposed measurement accuracy of the interference fringe period. In addition, the positioning accuracy of $\mathrm{x}$-dof is up to $6 \mathrm{~nm}$, which meets the dynamic performance requirements of scanning interference exposure process.

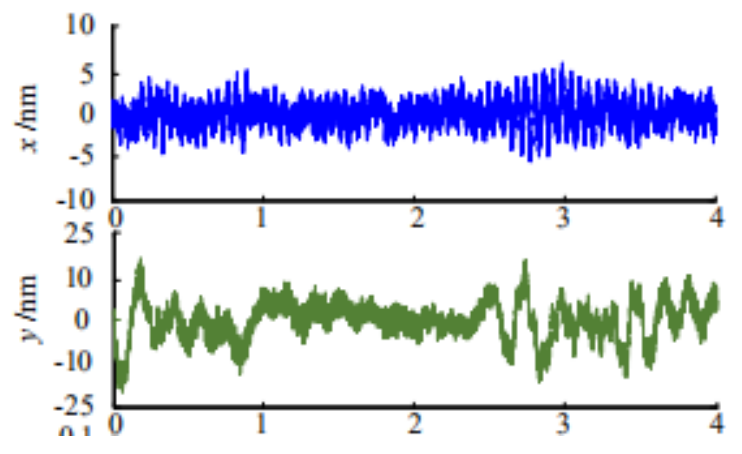

Figure 7 The positioning error of XY degree

\section{Conclusion}

In this paper, the optimization design method based on ANSYS is applied to the design of the air bearing of the precision work stage. First, the pressure distribution characteristics of the air bearing film are analyzed by using the finite element software. On this basis, the air bearing with high loading capacity and high stiffness is designed for the omni-directional preloading of the work stage. Finally, the XY DOF stage with vertical static stiffness of $103.7 \mathrm{~N} / \mu \mathrm{m}$, horizontal static stiffness of $213.6 \mathrm{~N} / \mu \mathrm{m}$ and bearing capacity of $602 \mathrm{~N}$ is designed. Based on PID and trap filter, a stable and high-precision control of the stage is realized by using grating encoder as the measurement feedback. The experiment shows that the position accuracy of $20 \mathrm{~nm}$ can be maintained stably, and the manufacturing of large-scale optical grating with nanometer precision can be guaranteed.

\section{References}

1. Yang S, Ding H, Li B. Scientific issues on Key Technologies of high-end manufacturing equipment [J]. Mechanical manufacturing and automation, 2011, 40 (001): I-5

2. Zhu Y, Yin W, Duan G. Research on ultra-precision stage of lithography machine [J]. Special equipment for electronic industry, 2004 (02): 26-28 + 45

3. Chen X D, Yu X Z, He X M, et al . Dynamic characteristic analysis of precision linear motor with air-bearing in optical lithography [J] .Chinese Journal of Mechanical Engineering, 2008, 21(2) : 17 -22 .

4. Cai T, Zhang M, Zhu Y, et al . Dynamic modeling and analysis of a 3-DOF ultra-precision positioning stage with air bearing [J] Procedia Engineering, 2011,16,264 -270 .

5. Zhang C, Liu Q. Development of linear motor high performance Worktable Based on static pressure air bearing guide rail $[\mathrm{J}]$. Mechanical science and technology, 2006,10 (10): 1212-1216

6. Chen P, Wang Y. Modeling and parameter optimization of precision air floating worktable system based on model center [J]. Modular machine tool and automatic processing technology,2014,4:2631 